\title{
Instability onset for submerged cylinders
}

\author{
Leo M. González, Esteban Ferrer \\ Universidad Politécnica de Madrid \\ Arco de la Victoria 4, 28040 Madrid, Spain \\ Juan M. Gimenez \\ Centro de Investigación de Métodos Computacionales (CIMEC) - UNL/CONICET \\ Santa Fe, Argentina
}

(Dated: November 26, 2018)

\begin{abstract}
This paper describes how the global stability of a circular cylinder is affected when submerged in a two-phase gravitational flow. The flow behavior is governed by both the Reynolds and the Froude number, while the depth of the cylinder has been varied to create different scenarios for the stability analysis. The baseflow obtained by the numerical solution of the 2D Navier-Stokes equations has been analyzed, and the first bifurcation (i.e. Hopf type) has been explored for different depths, Reynolds and Froude numbers. In addition to the typical vortex shedding instabilities associated to isolated cylinders, the presence of an interface between fluids creates new instabilities associated with the free surface that present more complex and deformed structures. According to the region of the parameter space studied here, two main causes of instabilities have been found: the ones provoked by vortex shedding on the cylinder wake (wake instabilities) at low Froude numbers and the ones produced by the free surface deformation (free surface instabilities) at high Froude numbers. When instabilities are related to vortex shedding, the critical Reynolds number and the frequency of the most unstable mode are comparable to the classical solution without free surface and gravity effects. In all cases, the shape of the most unstable mode is deformed and distorted according to the free surface location, while the critical Reynolds numbers and the frequency associated to the perturbation are both affected by the gravity and the free surface presence.
\end{abstract}

\section{INTRODUCTION}

The interaction between the viscous wake of a submerged object and the free surface position is a problem that deserves attention. For example, 1 justifies the study of this problem by its potential relevance for the remote sensing of the ocean surface from satellites, while [2, 3, outline its application to the design of offshore structures and vessels. It is well known that in the absence of free surface and gravity, the von Kármán vortex street generated by flow past an infinitely long circular cylinder produces a two dimensional time periodic flow for Reynolds numbers between approximately 47 and 189 [5] 8 . When gravity is added to the problem, the dynamics of the large-scale coherent structures around a circular cylinder in an open channel under very shallow and turbulent flow conditions where the bed friction significantly affects the wake structure is studied in 24. The complex wake created by an emergent cylinder with a large aspect ratio in a shallow open channel flow is studied experimentally using particle image velocimetry in [25]. Another relevant experimental free surface study, where the surface-height profile and vertical velocity distributions of a fully submerged hydrofoil were measured in [26]. This work focuses on the changes produced in the stability of this flow when the cylinder is submerged and a free surface separating two distinct fluids in the presence of gravity. In contrast, to the works referenced [24] and 25], here gravity acts orthogonal to the cylinder axis and the flow is laminar. Different 2D steady base- flows have been numerically studied and a linear global stability analysis has been performed in order to quantify the differences in the onset of the first instability when free surface and gravity are added to the problem. Previous authors performed stability analysis of idealized problems such as simple vortex structures 9, 10, and analytic shear flows [1]. To the authors knowledge, this constitutes the first study where a global stability analysis is performed on a Navier-Stokes computed solution in the presence of density discontinuities caused by the free surface. In order to gain insight about the physics of the interaction, the limits of steady solution where the velocity, the pressure and the free surface finally reach a stationary state are analyzed. The problem depends on two non-dimensional numbers, the Reynolds and the Froude numbers and one geometrical parameter, which is the cylinder depth (i.e. distance from the top of the cylinder to the free surface at rest). The classical single phase case when, in the absence of gravity, only one fluid is used has a very well studied solution and the steady separation bubble breaks its symmetry (i.e. becoming unstable) when the Reynolds number increases its value above the critical value $R e_{c}^{s p} \approx 47$ with non-dimensional frequency given by the Strouhal number $S t_{c}^{s p} \approx 0.11$ (both based on the cylinder diameter). For Reynolds numbers above this critical value, perturbations amplify to destabilize the separation bubble. In our study the Reynolds number has been increased from subcritical to supercritical values while the Froude number based on the cylinder diameter has been kept constant for three different cylin- 
der depths. Contrary to the unsubmerged case (no free surface), two causes could trigger the first instability of the submerged cylinder: typical vortex shedding instability or other free surface instabilities (i.e. wave breaking or secondary wave formation). In our study the range of parameters Reynolds, Froude and cylinder depth has been carefully selected such that different causes of instability appear. Free surface instabilities are dominant in some parts of the selected parameter space and their associated perturbation amplitudes can be distinguished from the typical vortex shedding instabilities (one phase). Rayleigh-Taylor instabilities are not expected considering that the heavier fluid is always placed at the bottom. In order to extend the flow analysis to those cases beyond the critical point, a Dynamic Mode Decomposition (DMD) 20, 21 technique, has been performed on the supercritical range. The latter analysis confirms that the frequencies associated to the most unstable mode are well captured through the linear stability analysis of the base flow obtained through frequency damping [19]. Similarly to the work presented by Triantafyllou and Dimas [1], the purpose here is to analyze the stability of the wake of floating two-dimensional objects. However, a few differences can be found between the two methodologies. First, in our case no analytic hypothesis of the flow or weakly parallel assumptions have been used for the baseflow construction, instead, the base flow has been computed solving the Navier-Stokes equations for both fluids. Second, no boundary conditions have been used for the free surface, and a scalar function, and the Volume of Fluid (VOF) function[11] has been included in the simulation to represent density and dynamic viscosity changes. Third, a two-dimensional global analysis has been performed, and consequently we have not assumed any harmonic dependance of the perturbation in the flow direction (i.e. parallel flow).

\section{METHODOLOGY}

The governing equations are the incompressible Navier-Stokes equations in laminar regime, which are supplemented with the conventional boundary conditions on solid and/or open boundaries. The computational domain $\Omega$ contains both fluids with different density and dynamic viscosity superposed one over the other. The first one is placed at the top of the domain (denoted by subscript 1) and the second at the bottom (subscript 2). Let us name their corresponding densities and kinematic viscosities as $\rho_{i}$ and $\nu_{i}(i=1,2)$, respectively, where $\rho_{2} \gg \rho_{1}$. The non dimensional governing equations, written in a Lagrangian framework, are:

$$
\begin{aligned}
& \nabla \cdot \mathbf{v}=0 \\
& \rho \frac{D \mathbf{v}}{D t}=-\nabla p+\frac{1}{R e} \nabla \cdot(\mu \nabla \mathbf{v})+\frac{\rho}{F r_{D}^{2}} \mathbf{u}_{\mathbf{g}}
\end{aligned}
$$

Here $D / D t, \mathbf{v}, p, \rho$ and $\mu$ are the material derivative, velocity, pressure, density and dynamic viscosity nondimensional variables and $\mathbf{u}_{\mathrm{g}}$ is a unitary vector parallel to the gravity force. The equations are computed in non-dimensional form using the cylinder diameter $D$, the inflow velocity $U$ and the density and dynamic viscosity of the bottom fluid such that pressure is nondimensionalized with $\rho_{2} U^{2}$. The Reynolds $R e=\frac{U D}{\nu_{2}}$ and Froude $\mathrm{Fr}=\frac{U}{\sqrt{g D}}$ numbers are defined using the properties of the bottom fluid. Following the classical theoretical framework used in this kind of problems, see 22 , in addition to equations (1) and (2), we have

$$
\frac{D \rho}{D t}=0
$$

which ensures that the density of every particle remains unchanged as we follow its motion. The VOF function $\phi$ is used to track the free surface, such that: $\phi=0$ in the fluid 1 and $\phi=1$ in the fluid 2. As usual, the free surface is represented by VOF function isosurface $\phi=0.5$. An efficient and accurate Particle Finite Element Method (PFEM-2) 12 has been used to numerically simulate the dynamics of the incompressible baseflows. It is important to remark that both density and dynamic viscosity are discontinuous functions that are accurately transported according to the techniques detailed in 12 .

The relation between the density and dynamic viscosity of the fluid and the VOF function is:

$$
\begin{aligned}
& \rho=(1-\phi) \rho_{1}+\phi \rho_{2}, \\
& \mu=(1-\phi) \mu_{1}+\phi \mu_{2},
\end{aligned}
$$

We choose a sufficiently large domain compared to the cylinder size to minimize undesirable boundary effects. The inflow and exit boundaries are located at $18 \mathrm{D}$ and 90D upstream and downstream of the cylinder, respectively. Side boundaries are located 20D apart from the central axis. A uniform inflow boundary condition, together with a natural boundary condition for the far field and outflow, are used for the velocity. No-slip boundary conditions are employed for cylinder walls. A Neumann boundary condition is imposed for the pressure in all boundaries except at the top where a fixed total pressure condition is imposed. Details are provided in FIG. 1. In order to avoid reflection of waves from outlet boundaries an absorption or relaxation zone is set in the last 30D of the domain. In this zone an explicit damping force to components of the velocity field in the direction of gravity is imposed to damp wave motion, and prevents reflections from the outlet boundary [23].

After a mesh convergence process based on the drag and lift forces, a final mesh has been retained. The mesh used for the baseflow simulations is a finite volume mesh that contains 32122 cells, the default mesh size is $0.7 \mathrm{D}$ while in the cylinder proximity and the free surface 
region, the mesh size has been refined to 0.01D. The global mesh and the mesh refinement near the cylinder are detailed in FIG. 2. This mesh was transformed into a P2-P1 finite element by cell division of the original finite volume mesh, generating a second mesh with 65834 triangles and 132542 quadratic nodes for the stability analysis computations.

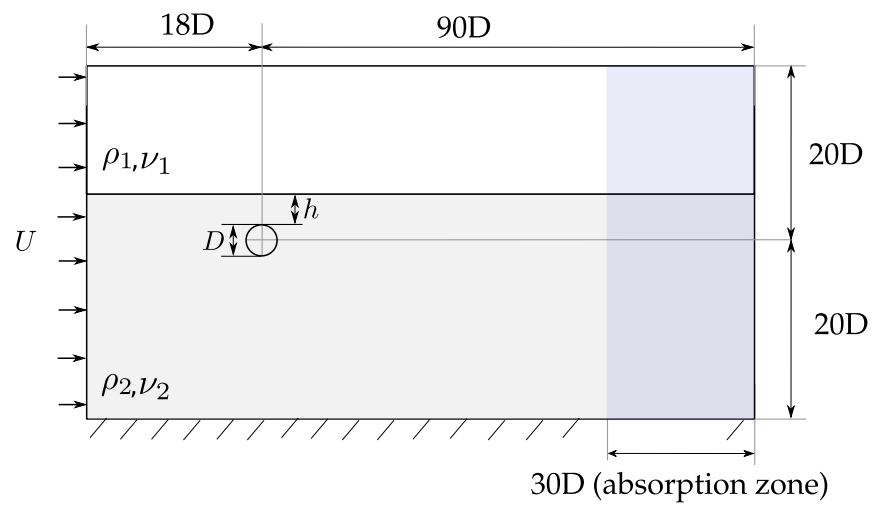

FIG. 1: Computational domain description for baseflow computation and subsequent stability analysis.

An accurate resolution of the free surface interface is crucial to extract stability information and hence special care is taken to resolve well this region such that the interface remains sharp along the time iterative solution. Large jumps of fluid density and dynamic viscosity across the interface should be correctly captured by the
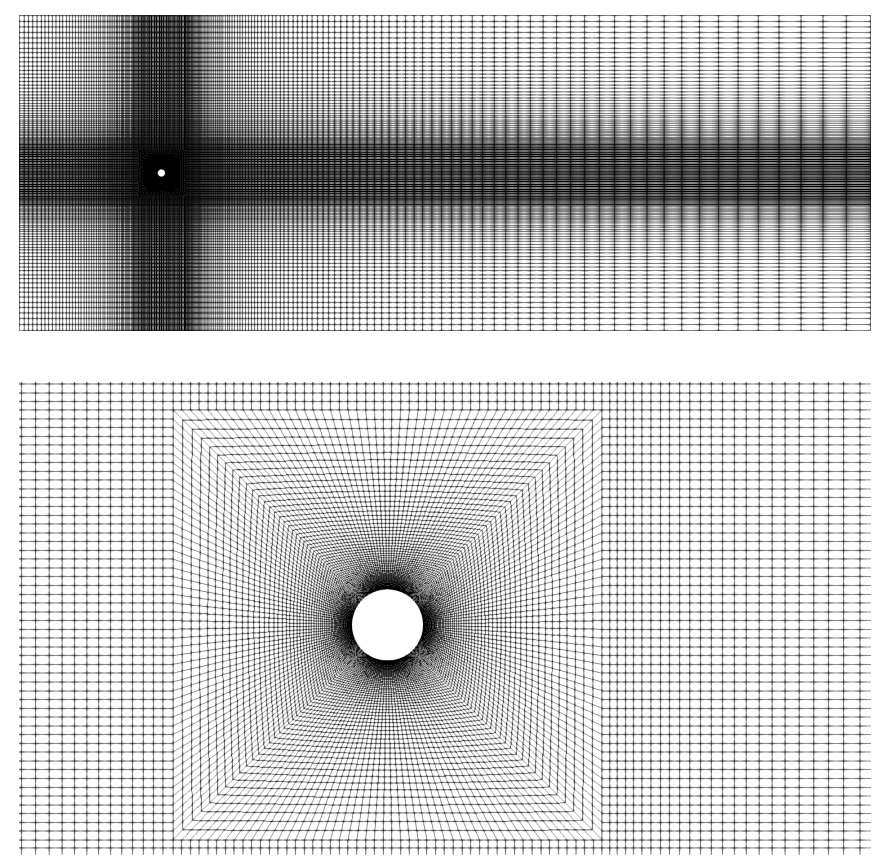

FIG. 2: Global (top) and zoomed view around the cylinder (bottom) of the mesh used for the baseflow computation and subsequent stability analysis. numerical algorithm in order to satisfy the momentum balance in the vicinity of the interface. Readers interested in more details of the PFEM-2 method and the enrichment technique used for the free surface definition may see [12.

The complete stability analysis requires two steps: during the first one, the two-dimensional Navier-Stokes equations are solved for the $R e$ and $F r$ values that reach a steady or periodic state $(\overline{\mathbf{v}}, \bar{p}, \bar{\phi})$ known as base flow. The equations for the baseflow read:

$$
\begin{aligned}
\bar{\rho} \frac{D \overline{\mathbf{v}}}{D t} & =-\nabla \bar{p}+\frac{1}{R e} \nabla \cdot(\bar{\mu} \nabla \overline{\mathbf{v}})+\frac{\bar{\rho}}{F r_{D}^{2}} \mathbf{u}_{\mathrm{g}} \\
\nabla \cdot \overline{\mathbf{v}} & =0, \\
\frac{D \bar{\phi}}{D t} & =0
\end{aligned}
$$

which are complemented with 4 and 5

In the problem solved in what follows, the component along the third spatial direction $(z)$ of the basic flow velocity vector is taken to be zero $\overline{\mathbf{v}}=(\bar{u}, \bar{v}, 0)$, and all components are taken to be independent of this spatial direction, $\frac{\partial}{\partial z}=0$. The consequence is that the linearized equations of motion defining the biglobal stability problem may be expressed by real operators. The basic flow is obtained by time-integration of system 6.8 starting from rest and evolving driven by the boundary conditions.

For the cases where the Reynolds number is above the critical value $R e>R e_{c}$ and no steady-state is obtained (e.g. periodic case) a last step is performed to obtain the baseflow. In those cases, an artificial steady-state is forced through a frequency damping technique 19. Some final tests are performed by DMD analysis of time varying flow without frequency damping, to confirm that the results obtained damping the frequencies that turn the flow to instability were correct (see Appendix).

Regardless of how the base flow is obtained, during the second step the base flow (steady or damped) is perturbed with small wave like ansatz for velocity and pressure such as:

$$
\mathbf{v}=\overline{\mathbf{v}}+\varepsilon \widehat{\mathbf{v}} \exp (\omega t) \quad p=\bar{p}+\varepsilon \widehat{p} \exp (\omega t),
$$

where $\varepsilon \ll 1$ and $\omega=\omega_{r}+i \omega_{i}$ is a complex number that contains the growth rate $\left(\omega_{r}\right)$ and the oscillation frequency $\left(\omega_{i}\right)$ of the perturbations. The stability analysis of the equations implies the linearization of the Navier-Stokes equations around a steady or damped flow (e.g. a frequency damping method is used to obtain a steady stated). This methodology has been extensively used by the authors and the results are presented in [14, 16], 17, 21], 15] and [18. Substituting into equations 1 1,3 , subtracting the basic flow equations 6 . 8. and linearizing, the equations for the perturbation quantities are obtained 


$$
\begin{gathered}
\nabla \cdot \widehat{\mathbf{v}}=0, \\
\bar{\rho} \frac{\partial \widehat{\mathbf{v}}}{\partial t}+\overline{\rho \mathbf{v}} \nabla \widehat{\mathbf{v}}+\bar{\rho} \widehat{\mathbf{v}} \nabla \overline{\mathbf{v}}+\widehat{\rho} \overline{\mathbf{v}} \nabla \overline{\mathbf{v}}=-\nabla \widehat{p}+ \\
\frac{1}{R e} \nabla \cdot(\bar{\mu} \nabla \widehat{\mathbf{v}}+\widehat{\mu} \nabla \overline{\mathbf{v}})+\frac{\widehat{\rho}}{F r_{D}^{2}} \mathbf{u}_{\mathbf{g}}, \\
\frac{\partial \widehat{\rho}}{\partial t}+\overline{\mathbf{v}} \nabla \widehat{\rho}+\bar{\rho} \nabla \widehat{\mathbf{v}}=0
\end{gathered}
$$

The system 10 12 is thus transformed into the generalized eigenvalue problem to determine the complex eigenvalue $\omega$ and eigenvector $\mathbf{X}$,

$$
A \cdot \mathbf{X}=-\omega B \cdot \mathbf{X}
$$

where $\mathbf{X}=(\widehat{\mathbf{v}}, \widehat{p}, \widehat{\rho})$ and the linear operators $A$ and $B$ are real. The process followed and the description of the operators $A$ and $B$ is detailed in [14, and implies the resolution of a large generalized eigenvalue problem by an iterative Arnoldi method.

The baseflow can be considered unstable when any of the growth rates $\omega_{r}$ are positive. Note that when the Navier-Stokes equations are solved and perturbed, terms that include the density gradients may become important and have to be taken into account in the proximity of the free surface. The boundary conditions used for the perturbed problem have been already described, with the exception of the inflow boundary condition where zero velocity perturbation is imposed. In addition, two important considerations should be remarked when the analysis is performed:

- For subcritical Reynolds numbers, the steady baseflow implies a final distribution of density and dynamic viscosity determined by the values of the VOF function.

- For supercritical cases, where the baseflow is unsteady, a frequency damping algorithm [19] is performed. For those supercritical cases, the linear global stability analysis is performed on an damped baseflow. Results are confirmed by an unsteady DMD analysis using no less than 10 snapshots per period (see Appendix ).

\section{RESULTS}

In all this work, the liquid (bottom fluid) density and dynamic viscosity are 100 times their respective top fluid value. The Froude number has been varied in the interval $F r \in[0.25,4]$ and three different water depth values were studied $h / D=0.55,1,2$. The bifurcation onset corresponding to the critical Reynolds number, $R e_{c}$, has always been found in the range $R e=[10,60]$.

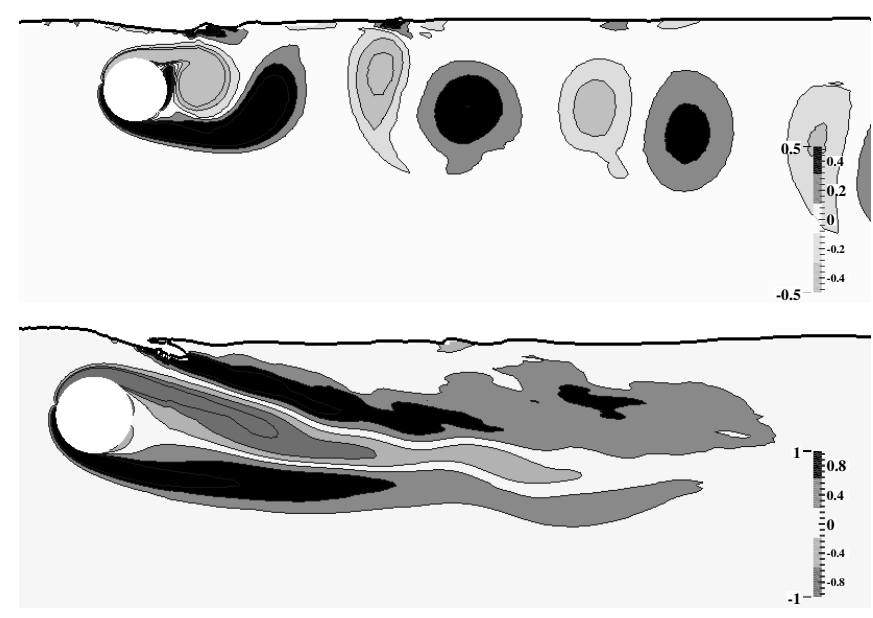

FIG. 3: Vorticity fields for depth of $h / d=0.55$, $R e=180$ and for Froude numbers of (top) $\mathrm{Fr}=0.3$, (bottom) $F r=0.6$. Positive vorticity is shown as dark grey and negative vorticity as light grey.

\section{Validation}

Despite the Reynolds number difference, the free surface elevation at the top of the cylinder is similar to the one presented by Bouscasse 3] and Reichl 2. For the sake of validation, the global drag and lift forces have been computed and compared against previous results 3 , 4] at the more studied conditions $R e=180, h / D=$ 0.55 and different Froude numbers. Results show good agreement, see FIG. 4. In FIG. 3, two vorticity field snapshots at $R e=180$ and $t=100$ at two different Froude numbers $F r=0.3$ and $F r=0.6$ are shown, as can be observed the free surface position and vorticity wake is very similar to the results presented by Bouscasse 4.

\section{Stability analysis}

Baseflow computation.

We begin by assessing the free surface deformation when varying the Froude number at a fixed Reynolds $R e=40$. Results are summarized in FIG. 5, where it becomes apparent that increasing the Froude number leads to longer deformations. Furthermore, an increase in Fr also results in a higher elevation at the cylinder position, suggesting that low Froude numbers lead to a narrow contraction between cylinder top and free surface and a consequent locally accelerated flow. The shape of the free surface also modifies locally the flow direction, introducing larger vertical flow components as the Froude number increases. Overall the free surface 


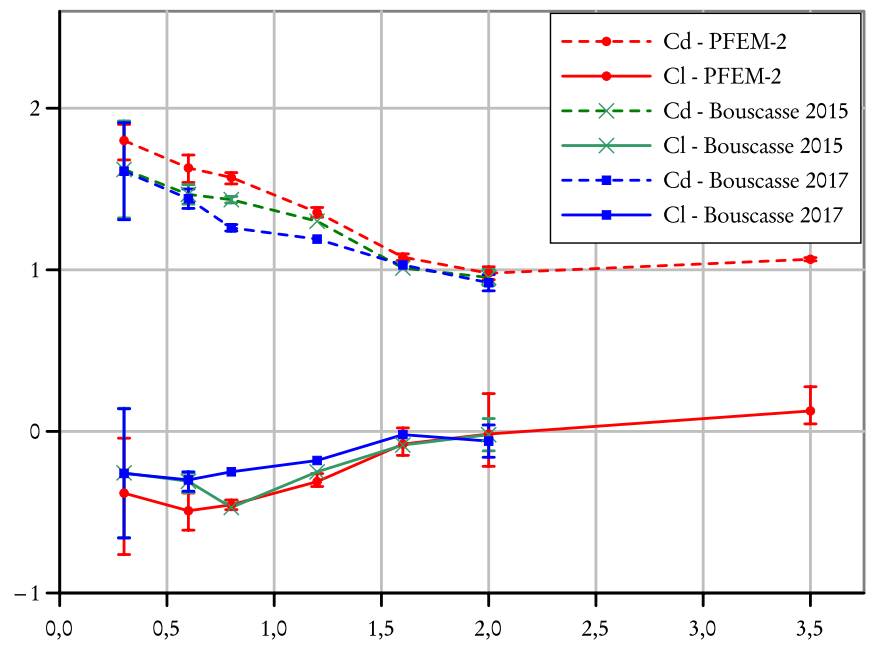

FIG. 4: Drag and lift coefficients calculated with PFEM-2 at $R e=180$ and $h / D=0.55$ for different $F r$ numbers, comparison with Bouscasse [3, 4].
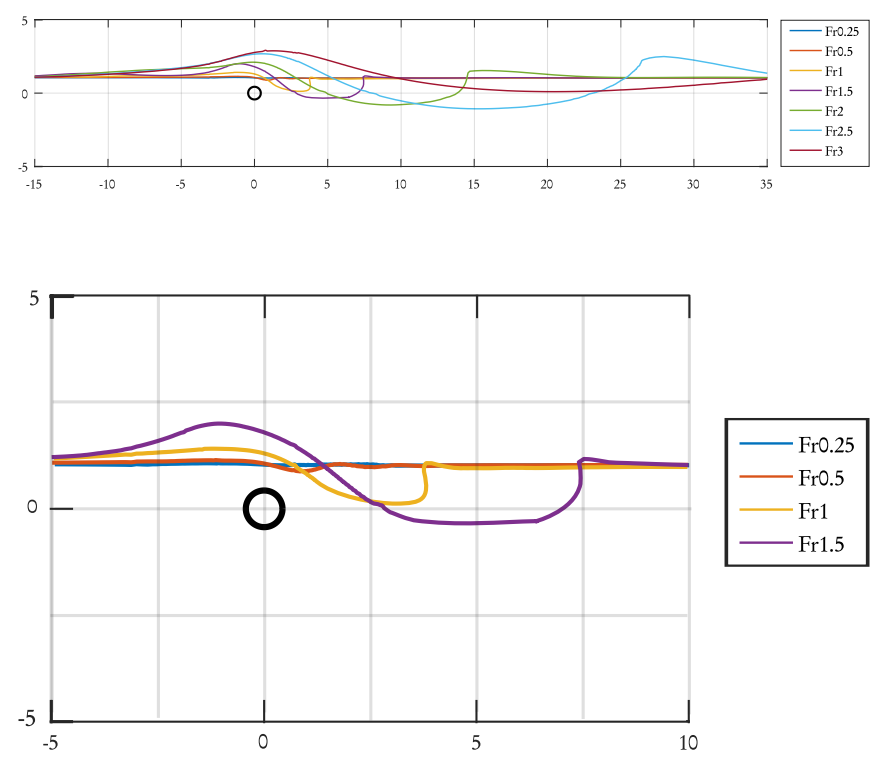

FIG. 5: Top: free surface position at $R e=40$ and $h / D=0.55$ for different Froude numbers. Bottom: zoomed view around the cylinder.

deformation provides base-flows that are substantially different when the Froude number is varied. In addition, the cylinder depth $h / D$ and the Reynolds number provide two additional parameters that modulate the shape of the base flow upon which the stability analysis is performed. An example is provided in FIG. 6, where the horizontal and vertical velocity components of the baseflow (including the free surface location) are shown for $R e=45, F r=3$ and $h / D=0.55$. The onset of instability is hence controlled by these parameters and quantified in following sections.
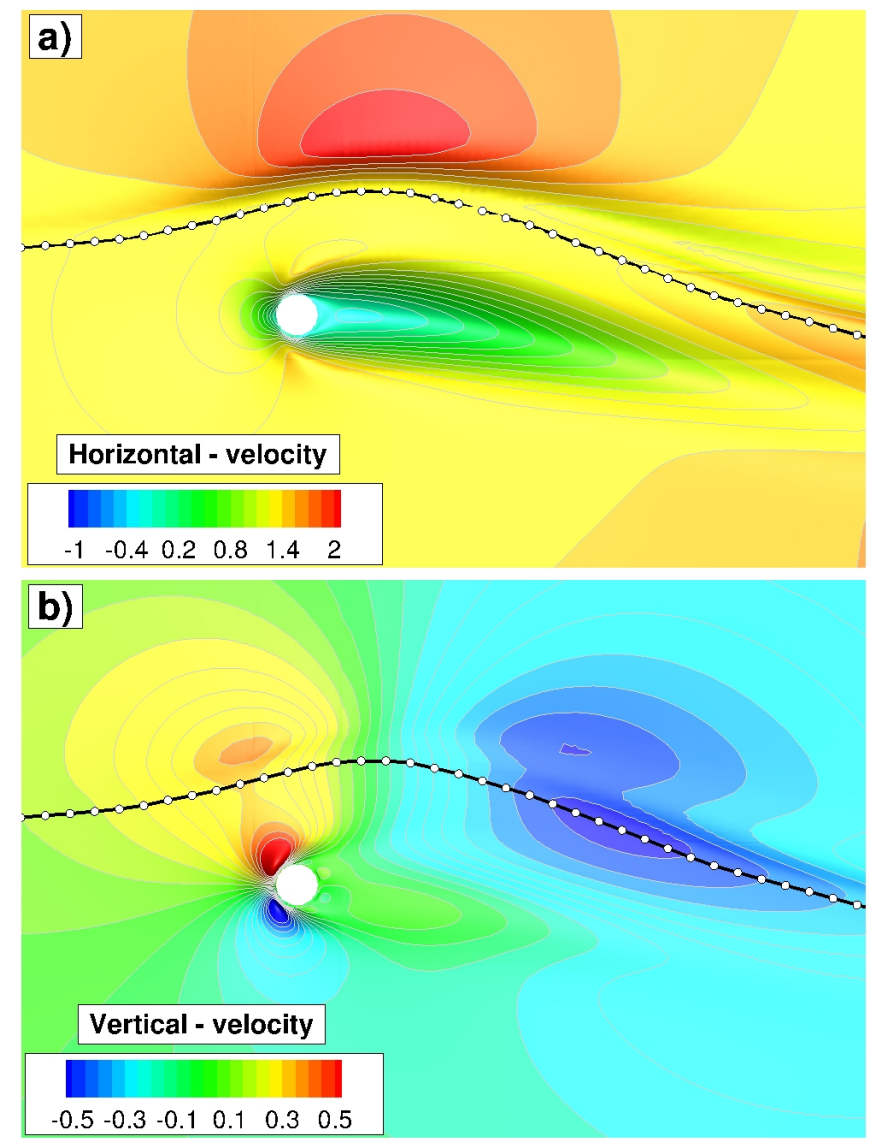

FIG. 6: Steady state velocity field for $R e=45$, $h / D=0.55$ and $F r=3$. The black line corresponds to the free surface location.

\section{Hopf bifurcation.}

Locating the cylinder under a deforming free surface results in a complex flow. In this case, the leading instability controlling the onset of unsteady flow (i.e. Hopf bifurcation) can be associated to two distinct physical phenomena. For some parameters, the classic wake instability (i.e. vortex shedding when the Reynolds number approximates its critical value $R e_{c}^{s p}$ [5]) that appears also in one-phase flows $(F r=\infty)$ dominates, whilst in other situations free surface instabilities associated to the interface deformation dominate. Depending on the values of the three parameters involved in this study: Froude, Reynolds and depth, vortex shedding or free-surface deformation dominates the onset of unsteadiness. Let us start the study presenting the different types of instabilities that have been found according to the mode shape and depending on the value of the parameters.

First, a wake instability dominates at high Froude numbers $\mathrm{Fr}>3$, these modes present associated 

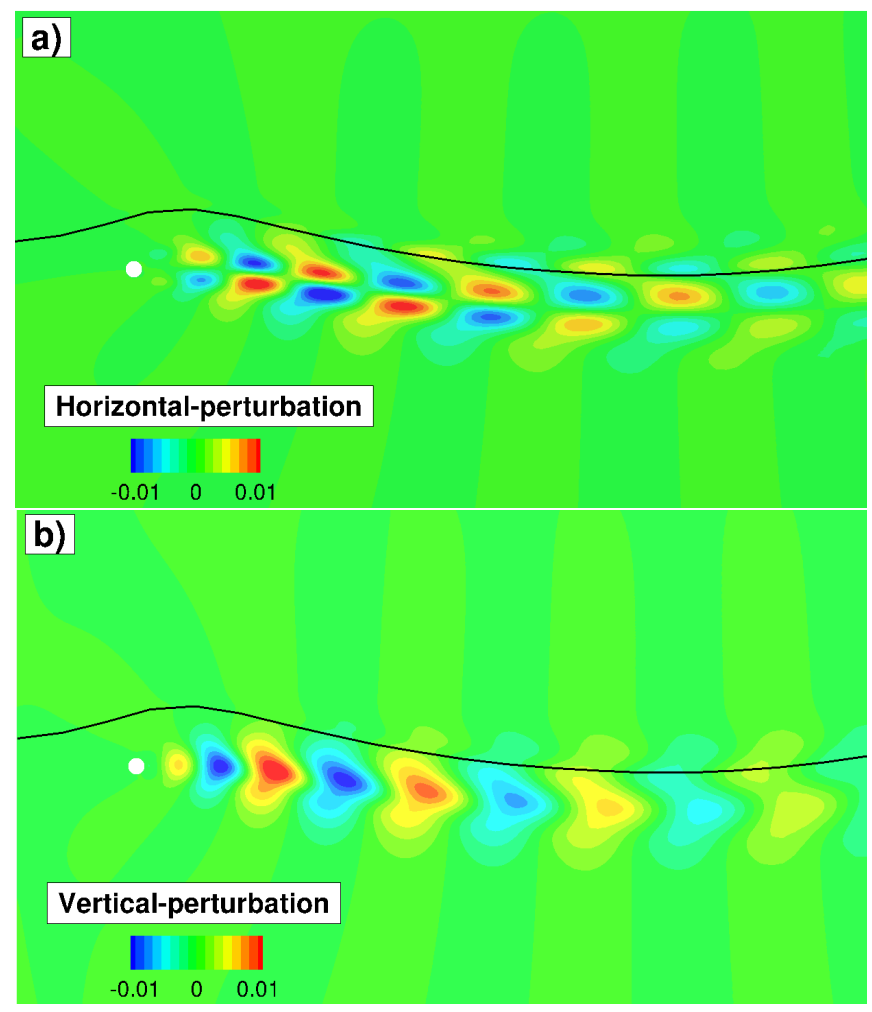

FIG. 7: Example of the shape of a deformed vortex shedding mode. Horizontal(top) and vertical(bottom) components of the velocity perturbation for $\mathrm{Fr}=3.5$,

$$
R e=50 \text { and } h / D=0.55
$$

frequencies which are not far from the classical vortex shedding frequency $S t_{c} \sim 0.11$ which is equivalent to the critical value $\omega_{i}^{c} \sim 0.73$. This most unstable mode can be visually identified by comparison to the one obtained for single phase flow $\mathrm{Fr} \rightarrow \infty$, with the only difference that the vortex shedding pattern that appears immediately behind the cylinder is deformed and modulated by the free surface shape. As vortex shedding is the dominant instability cause, the frequency of the most unstable mode remains close to $\omega_{i}^{c}$. A typical example of this kind of mode is found at $F r=3.5$ for the three values of the parameter $h / D$, see the components of the velocity perturbation amplitude in FIG. 7 when $h / D=0.55$ the critical Reynolds number is found at $R e_{c} \approx 50$.

A second type of instability is found when free surface deforms a few diameters away of the cylinder. This instability is generally found at intermediate Froude numbers $2.5<F r<1.5$, and the frequency associated to this kind is lower compared to the reference single phase one $\omega_{i}^{c}$. The shape of the most unstable mode is clearly associated to the free surface deformation and is found behind the cylinder, but far from the cylinder near-wake. See, for example, the velocity perturbation in FIG. 8 for $F r=2, R e=30$ and $h / D=0.55$.
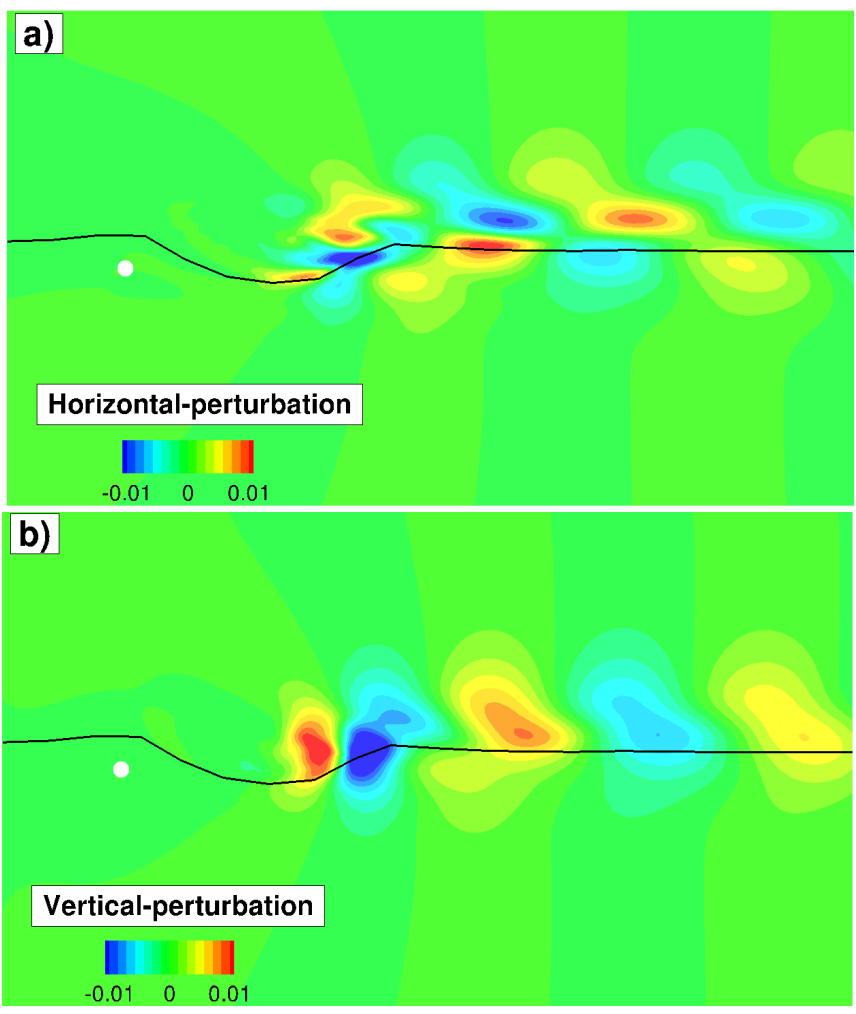

FIG. 8: Example of the shape of a free surface mode. Horizontal(top) and vertical(bottom) components of the perturbation for $F r=2, R e=30$ and $h / D=0.55$

Other types of instabilities that deserve attention are also found; a particular kind of free surface instability has been exclusively found at $F r=1$ and $h / D=0.55$, due to the contraction created between the cylinder and the free surface (blocked flow). This confined instability is found close to the free surface cylinder area, and has negligible frequency, see FIG. 9 for $R e=60$. It should be also mentioned, that in some situations the instability found is a combination of the two types of instabilities, wake and free surface, described before. Then, the mode amplitude presents a vortex shedding shape that propagates in both top and bottom fluids, this kind of instability appears at low Froude numbers $\mathrm{Fr}<1.5$ and the frequencies associated are close to the classical one due to the vortex shedding component, see FIG. 10

The evolutions of the growth/damping rates and the angular frequencies of the resulting most unstable mode are shown in FIG. 11, for various Reynolds and depths $h / D$, for three representative Froude numbers: $F r=3.5$, $F r=2$ and $F r=1$. The case without free surface $F r, h / D \rightarrow \infty$ is included as reference. The most unstable mode moves towards the unstable region $\omega_{r}>0$ when the Reynolds number is increased, similarly to what happens in the absence of free surface. For the highest Froude number, $\mathrm{Fr}=3.5$, the growth rate curves are 

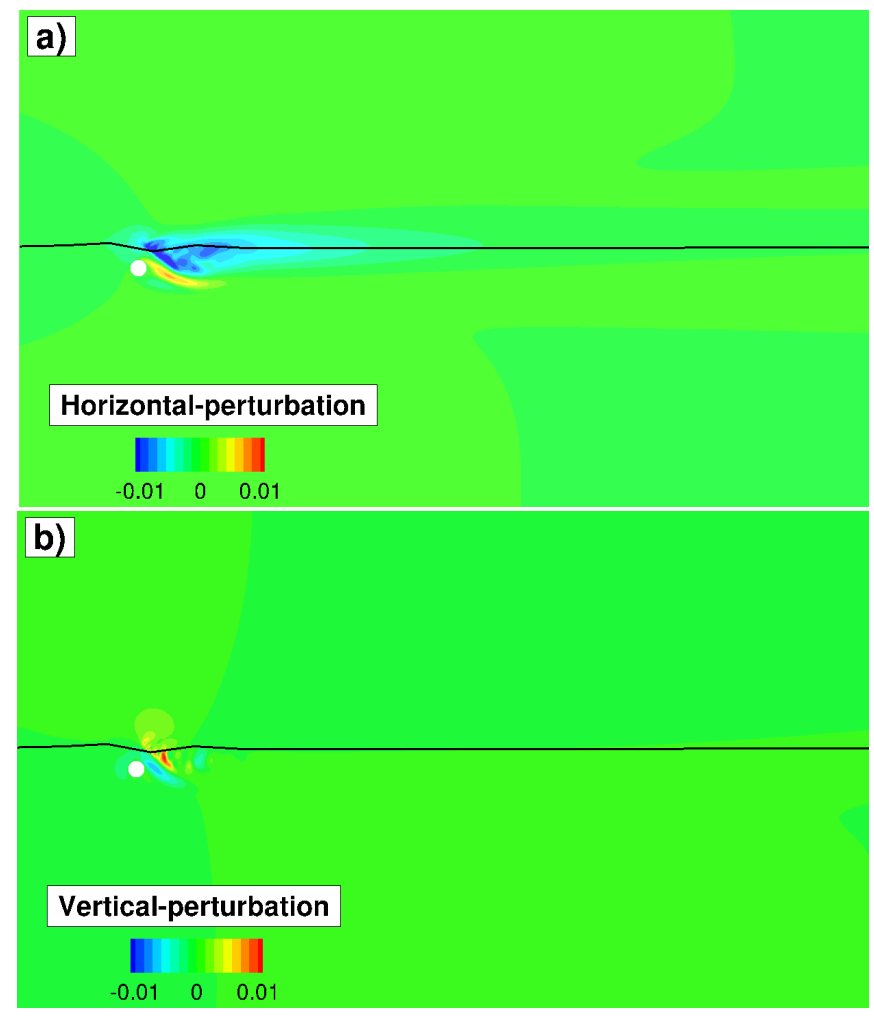

FIG. 9: Particular case of a stationary free surface mode when the interface deformation is very close to the cylinder. Horizontal(top) and vertical(bottom) components of the perturbation for $F r=1, R e=60$ and $h / D=0.55$

close to each other for all depths, which corresponds to the classic cylinder wake instability and not being caused by the free surface proximity to the cylinder. This is in good agreement with the mode presented in FIG. 7 for $F r=3.5$ and $h / D=0.55$, as a typical example of deformed (and modulated) vortex shedding instability. Furthermore, the frequency and the critical Reynolds numbers are not far from the ones presented for the single phase case, which is the canonical vortex shedding instability. When the Froude number decreases to $F r=2$, both the critical Reynolds number and the frequency decrease when compared to the single phase flow, meaning that in this circumstance the instability is not related to the vortex shedding and now depends on the free surface deformation and the cylinder depth. In fact, the lowest critical Reynolds numbers are found at $F r=2$ in particular for small depth $h / D$ values, being $R e \approx 20$ when $h / D \leq 1$. For the particular case $F r=1$ and minimum depth $h / D=0.55$, a zero frequency mode is found which agrees with the confined mode shape, see FIG. 9. If the depth is increased, the instability becomes a vortex shedding type, having frequencies and growth rates not far from the single fluid case. Similar to the
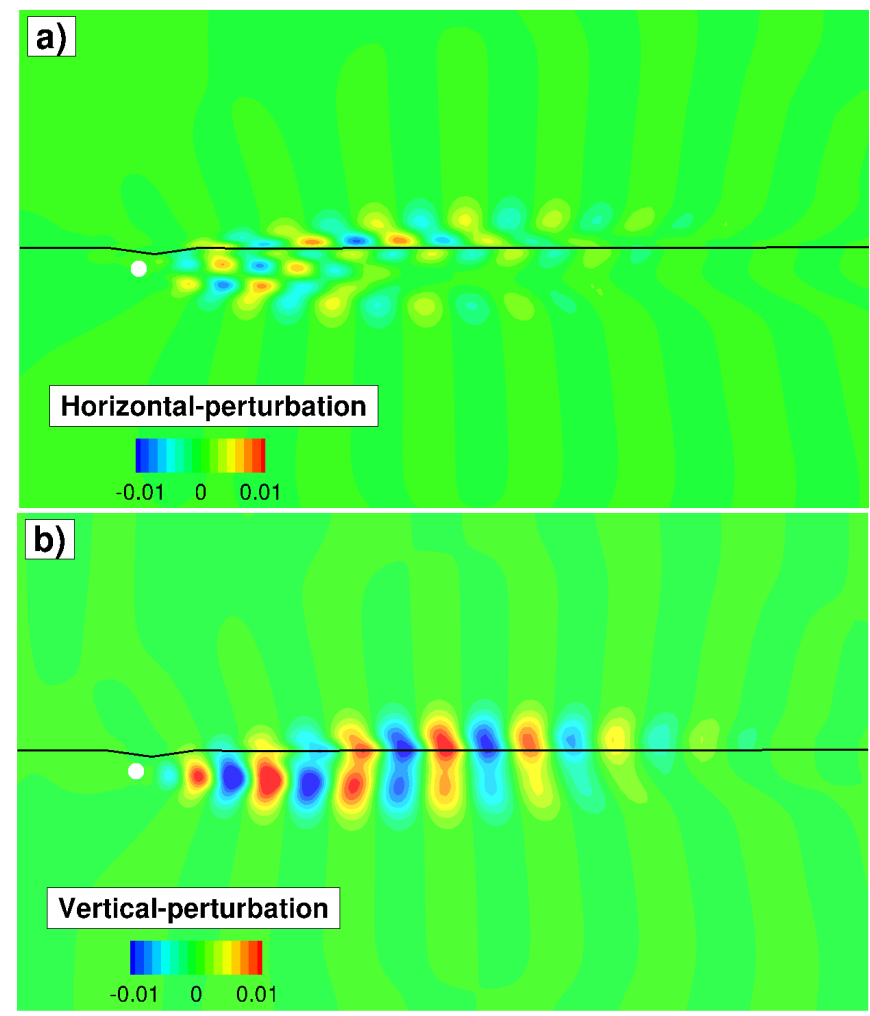

FIG. 10: Example of the shape of a combined vortex shedding-free-surface mode. Horizontal(top) and vertical(bottom) components of the perturbation for $F r=0.5, R e=60$ and $h / D=0.55$

$F r=2$ case, highest depth implies enhanced stability, consequently the critical Reynolds increases when $h / D$ increases. Comparing the single phase case $(h / D=\infty$ and $\mathrm{Fr} \rightarrow \infty$, red lines in FIG. 11) to the most similar case analyzed (maximum Froude number $F r=3.5$ and maximum depth $h / D=2$ ), we found that the critical Reynolds number is lower, suggesting that when the wake causes the instability the free surface presence has a stabilizing effect. This provides a first evidence that only when wake instabilities are responsible of the Hopf bifurcation, the free surface presence increases the stability of the flow, however this statement cannot be generalized to other free surface instabilities.

Additionally, at the bottom picture of FIG. 11, we can appreciate that at high Froude numbers $(F r=3.5)$ which present a vortex shedding instability, its angular frequency is affected by the submerged depth, and $\omega_{i}$ decreases when the ratio $h / D$ decreases. As $h / D$ is increased, the frequency tends to the one obtained when a single phase is considered. Therefore, it may be argued that for high Froude numbers and non-dimensional depths larger than $2(h / D \geq 2)$, the free surface presence does not affect significantly the perturbation frequency being close to $\omega_{c}$. Those frequency values are reduced for 

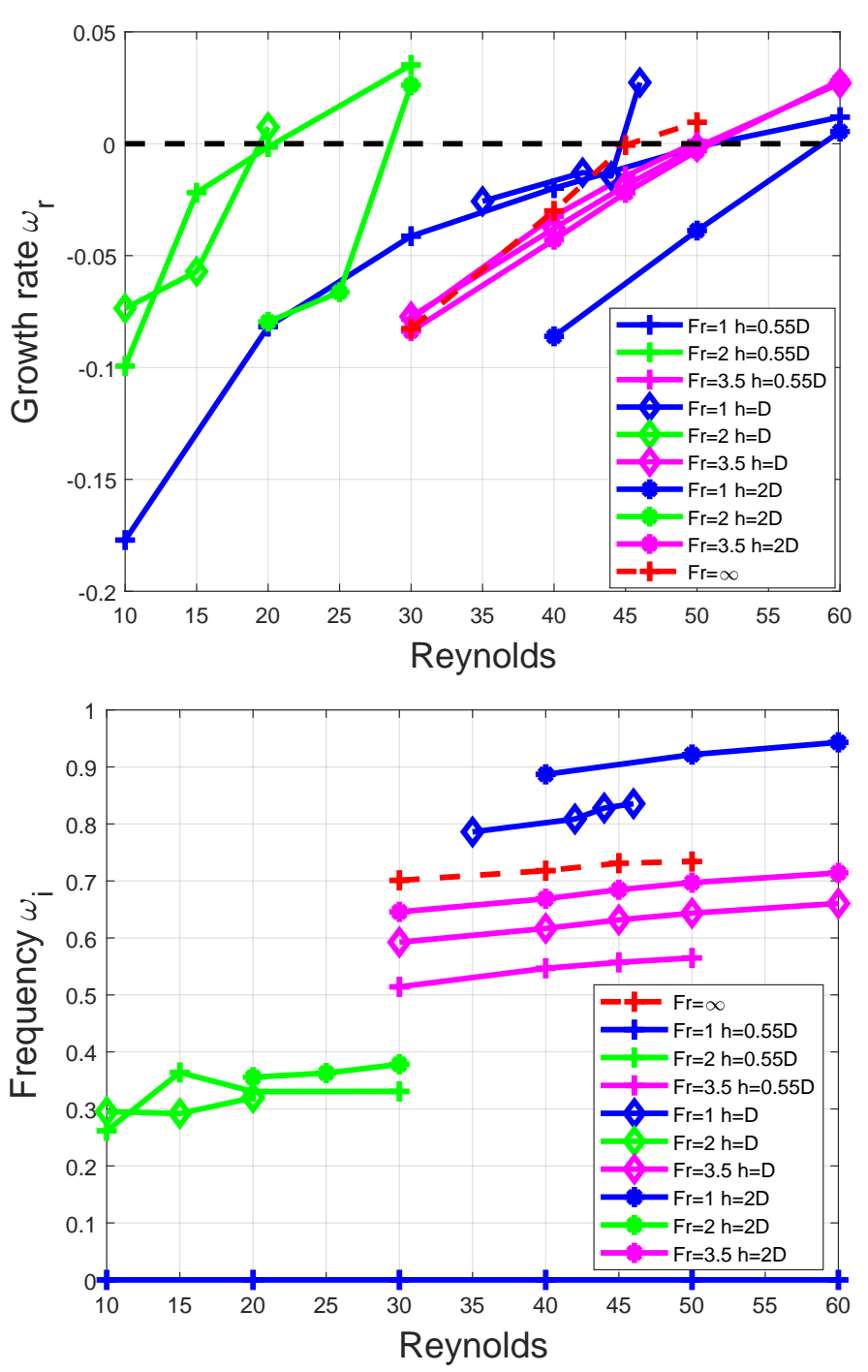

FIG. 11: Growth rate and frequency versus $R e$ number for representative Froude numbers $F r=1,2,3.5$ and all depth. The classical results of the Hopf bifurcation of the isolated cylinder problem without free surface and no gravity has also been added (red line), where it can

be observed that $R e_{c}=47$ and $\omega_{c}=0.7414$ are the expected critical values.

smaller depths $h / D<2$. The mode corresponding to this leading unstable mode for the case $R e=45, F r=3$ and $h / D=0.55$ is depicted in FIG. 12 , where the shape of the mode is visibly influenced by the free surface modulation.

In FIG. 15 the growth rate is plotted against the Reynolds number for different Froude numbers, when the depth $h / D=0.55$ is kept constant. We also represented in red the single phase case for comparison. We observe that according to the type of inherent instability the curves are clustered together. As we explained before, if the instabilities are purely or partially caused by some
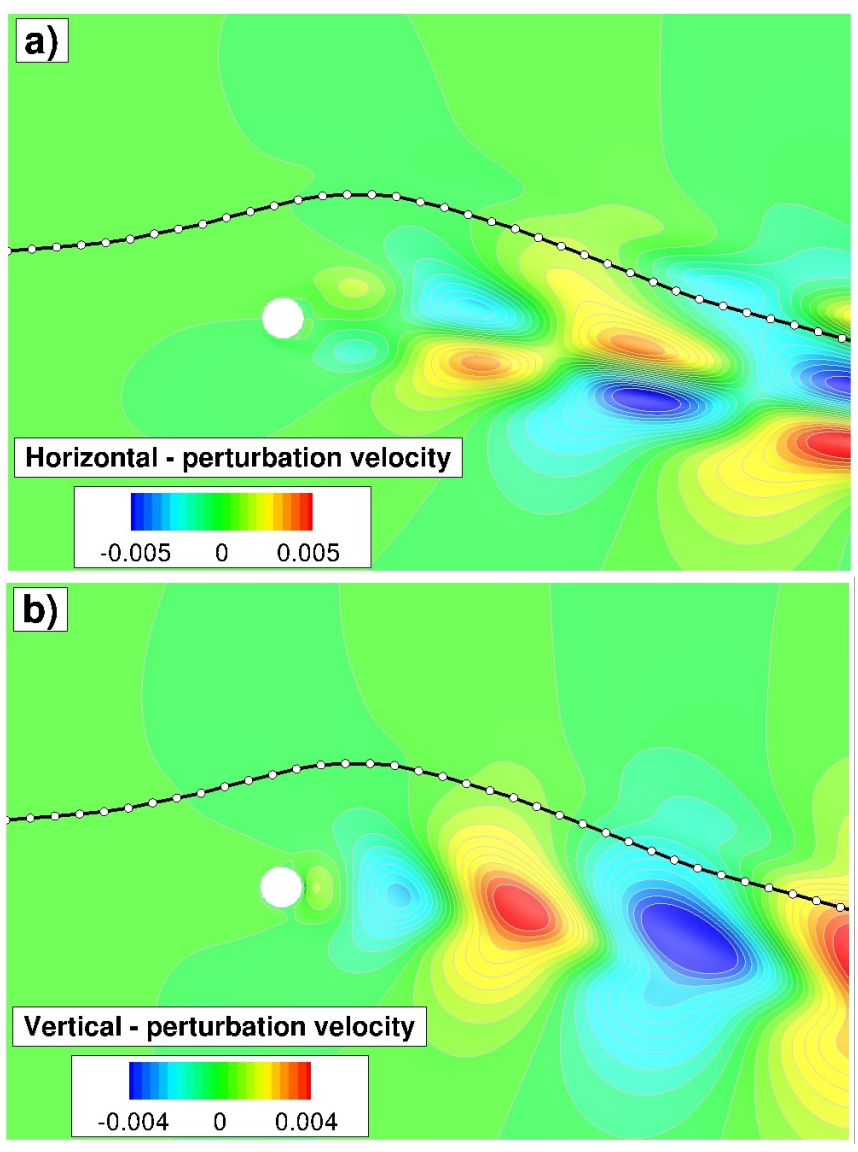

FIG. 12: Unstable mode (real part) for the case $R e=45 \mathrm{Fr}=3$ and $h / D=0.55$

kind of wake phenomena, the critical Reynolds number is close to the single phase critical value $\left(R e_{c}^{s p}\right)$. Vortex shedding occurs when $F r \geq 3$ and $F r \leq 0.5$, whilst for $2.5 \geq F r \geq 1.5$, free surface instabilities dominate. In the bottom part of FIG. 15, the angular frequency associated with the less stable mode is also represented versus the Reynolds number for different values of the Froude parameter. We can observe that using the red line corresponding to the single frequency case $\omega_{i}^{c}$ as reference, the frequencies of the perturbation modes are not far from this value. Namely, $\omega_{i}$ approaches $\omega_{i}^{c}$ when vortex shedding causes the instability, while the modes corresponding to free surface clearly present lower frequencies.

As the steady baseflow can be instabilized by two different causes: either the cylinder wake or the excessive free surface deformation. We should take into account that FIGS. 11 and 15 just presents the most unstable mode without attending to the cause, which can change depending on the Reynolds number. Consequently, the most unstable mode in a particular Reynolds range could be caused by the cylinder wake, but when the Reynolds number increases, free surface deformation modes might grow quicker and finally cause the instability or viceversa. 




FIG. 13: Variation of the critical Reynolds number $R e_{c}$ as a function of the Froude number $\mathrm{Fr}$ for different depths $h / D$. The critical Reynolds number for the single phase flow $R e_{c}^{s p}$ has been added as reference in red dashed line.

This mode competition is what causes the slope change in the curves. In this analysis, it is the mode shape what should give some light into the physical interpretation of the final cause of the instability when the instability limit $\left(\omega_{r}=0\right)$ is crossed.

FIG. 13 presents the variation of the critical Reynolds number $R e_{c}$ as a function of Froude number $F r$ for different depths $h / D$. This figure presents a critical Froude range around $\mathrm{Fr} \sim 2-2.5$ where the critical Reynolds number is minimized, this tendency is followed by the three depths studied. For the highest Froude numbers, the curves tend to approach the critical Reynolds number obtained for the single phase flow $R e_{c}^{s p}$, also represented in the figure.

For completeness, we include a summary of the results in FIG. 14. The figure includes the different types of instabilities, for varying Froude numbers and depths.

\section{CONCLUSIONS}

This work has presented for the first time global stability analysis results for a submerged circular cylinder under the modulation of a steady free surface. The stability of this particular cylinder is therefore affected by the free surface position relative to the cylinder center, the Reynolds number and the Froude number. A framework to analyze the Hopf bifurcation of this two-phase flow has been developed performing a global stability analysis for particular steady or quasi steady solutions. These results have shown that different

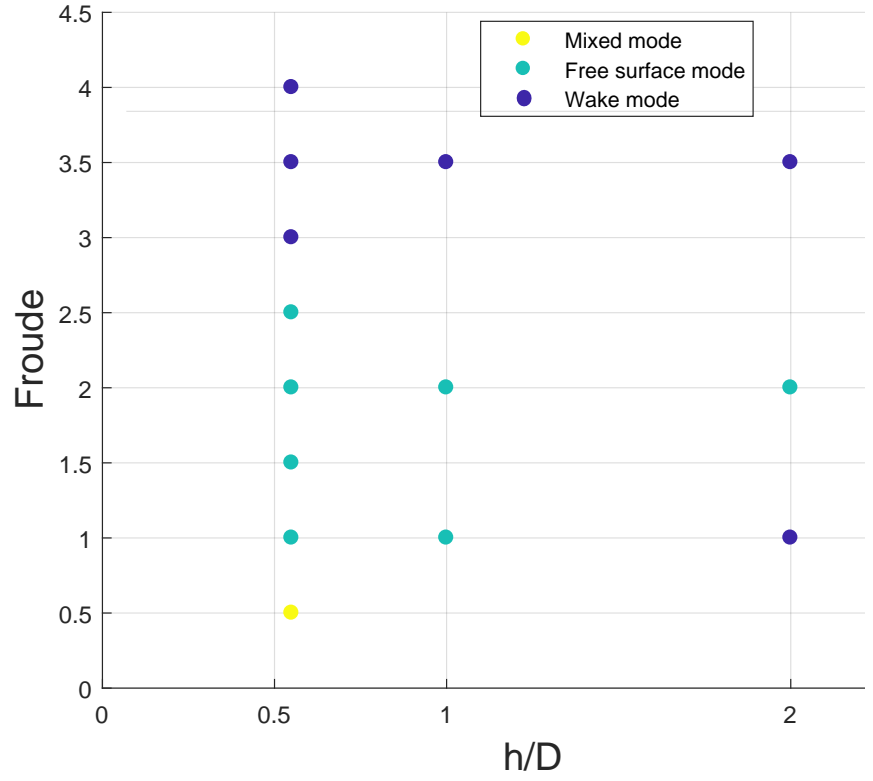

FIG. 14: Global representation of the types of instabilities found in our parameter space.

scenarios could appear depending on the parameter values and relative free surface position, as consequence besides the onset of wake instabilities associated to the von Kármán vortex street that generally appear when cylinders are studied, other unstable modes associated to the free surface interface also modify the flow stability. Modes that combine both phenomena and singularities such as stationary modes may also appear in the parameter space studied. As expected, the shape of the most unstable mode is always deformed and distorted due to the free surface presence. We conclude that, depending on the kind of instability, the critical Reynolds number can change severely, and the presence of free surface does not always enhances stabilization. In addition, the free surface has shown to modify the frequency associated to the less stable perturbations. The global scenario shows that using the single phase critical Reynolds number and critical frequency as reference, two high frequency regions appear associated to low and high Froude numbers that involve wake instability, and an intermediate interval of Froude numbers where the instabilities are associated to the free surface.

\section{ACKNOWLEDGEMENTS}

This work was supported by the Technical University of Madrid by the project (PID) "Combination of Eulerian and Lagrangian methodologies for the solution of free surface flows." 


\section{APPENDIX: DMD CONFIRMATION FOR SUPERCRITICAL REGIMES.}

We explore the frequency variations in the supercritical regime. This happens when the Reynolds number range is extended to larger Reynolds numbers, $R e>R e_{c}$, and frequency damped baseflows are used for the analysis. To confirm the results obtained by the (frequency damped) linear stability analysis, an unsteady DMD analysis has been also performed for $F r=3$ and two supercritical Reynolds numbers $R e=60$ and $R e=70$ for all cylinder depths. FIG. 16 compares the critical frequencies for the DMD analysis and the global stability analysis results obtained from the analysis of damped baseflows. It can be observed that the values of the frequencies agree well. In all cases the leading frequency is attributed to the vortex shedding instability in the cylinder wake, typically found at $F r=3$.

merlin.mbs apsrev4-1.bst 2010-07-25 4.21a (PWD, AO, DPC) hacked Control: key (0) Control: author (8) initials jnrlst Control: editor formatted (1) identically to author Control: production of article title (-1) disabled Control: page (0) single Control: year (1) truncated Control: production of eprint (0) enabled

[1] G. S. Triantafyllou and A. A.Dimas, Interaction of twodimensional separated flows with a free surface at low Froude numbers. Physics of Fluids A, 1,1989

[2] P. Reichl, K. Hourigan and M. Thompson, Flow past a cylinder close to a free surface. Journal of Fluid Mechanics, 533:269-296,2005

[3] B. Bouscasse, A. Colagrossi, A. Souto-Iglesias and S.Marrone, High Froude Number Viscous Flow Past a Circular Cylinder. International Conference on Ocean, Offshore and Arctic Engineering,2014

[4] B. Bouscasse, A. Colagrossi, A. Souto-Iglesias and S.Marrone, SPH modelling of viscous flow past a circular cylinder interacting with a free surface. Computers and Fluids, 146:190-212,2017

[5] C. P. Jackson, A finite-element study of the onset of vortex shedding in flow past variously shaped bodies. Journal of Fluid Mechanics,182:23-45,1987

[6] M. Provansal, C. Mathis and L. Boyer, Bénardvon Kármán instability: transient and forced regimes. Journal of Fluid Mechanics,182:1-22,1987

[7] C. H. K. Williamson, Defining a universal and continuous Strouhal-Reynolds number relationship for the laminar vortex shedding of a circular cylinder. Physics of Fluids 31 (1988).

[8] G. Miller and C. Williamson, Control of threedimensional phase dynamics in a cylinder wake. Experiments in Fluids 18, 1-2:26-35 (1994).
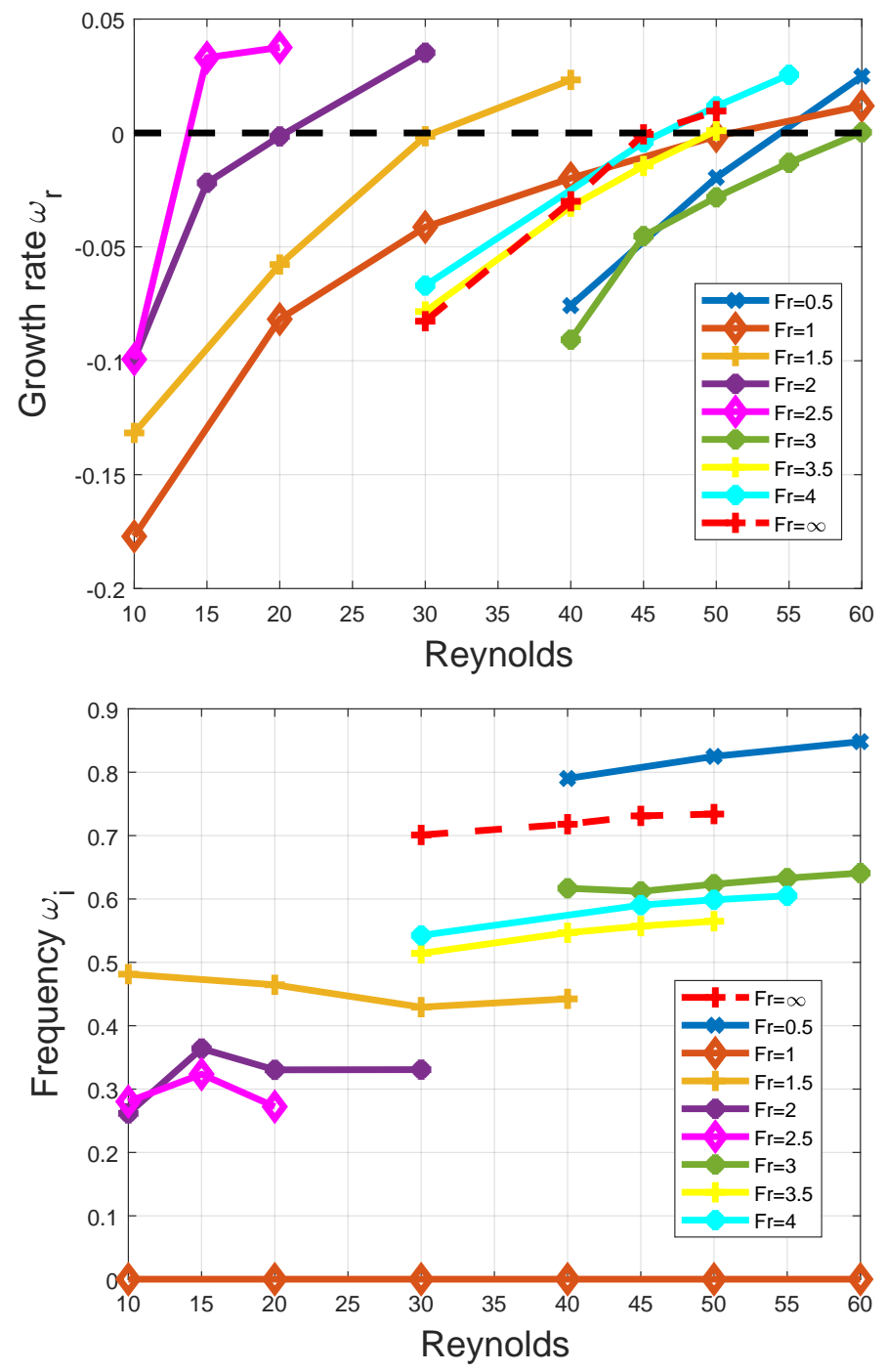

FIG. 15: Dependance of the growth rate and frequency on the Reynolds number of the most unstable mode for different Froude values at $h / D=0.55$.

[9] S. Ohring and H. J. Lugt, Interaction of a viscous vortex pair with a free surface. Journal of Fluid Mechanics 227, 227:47-70 (1991).

[10] J. Fontane and L. Joly, The stability of the variabledensity Kelvin-Helmholtz billow. Journal of Fluid Mechanics 612, 237 (2008).

[11] C. W. Hirt and B. D. Nichols, Volume of Fluid (VOF) Method for the Dynamics of Free Boundaries. Journal of Computational Physics 39, 201 (1981).

[12] J. Gimenez and L. González, An extended validation of the last generation of particle finite element method for free surface flows. Journal of Computational Physics 284, 186 (2015).

[13] D. Sipp and A. Lebedev, Global stability of base and mean flows: a general approach and its applications to cylinder and open cavity flows. Journal of Fluid Mechanics 593, 333 (2007). 


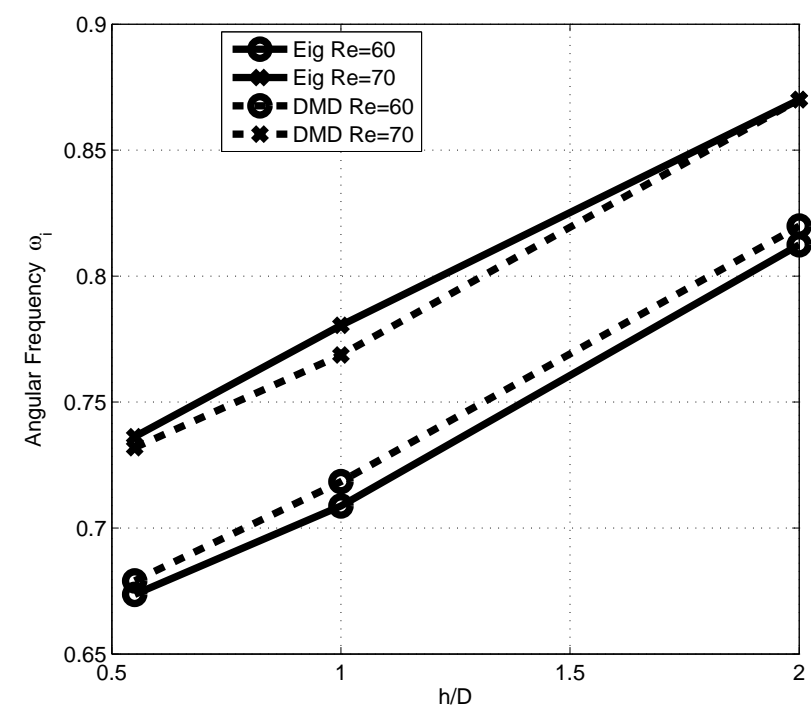

FIG. 16: Critical frequencies for varying cylinder depths and two Reynolds numbers at $F r=3$ : global analysis of frequency damped baseflows and unsteady DMD analysis.

[14] L. González and E. Ferrer, H.R. Diaz-Ojeda, Onset of three-dimensional flow instabilities in lid-driven circular cavities. Physics of Fluids 29, 064102 (2017).

[15] L. González and V. Theofilis and R. Gómez-Blanco, Finite-element numerical methods for viscous incompressible BiGlobal linear instability analysis on unstructured meshes. AIAA Journal 45, 840 (2007).

[16] L. González and R. Gómez-Blanco and V. Theofilis , Eigenmodes of a Counter-Rotating Vortex Dipole. AIAA
Journal 57, 840 (2008).

[17] L. M. Gonzalez and M. Ahmed and J. Kuhnen and H. C. Kuhlmann and V. Theofilis, Three-dimensional flow instability in a lid-driven isosceles triangular cavity. Journal of Fluid Mechanics 675, 369-396 (2011).

[18] L. M. González and V.Theofilis and S.Sherwin , Highorder methods for the numerical solution of the BiGlobal linear stability eigenvalue problem in complex geometries Int. J. Numer. Meth. Fluids 65, 923-952 (2011).

[19] B. E. Jordi, C. J. Cotter, and S. J. Sherwin, An adaptive selective frequency damping method. Physics of Fluids (1994-present) 27, 094104 (2015).

[20] P. Schmid, Dynamic mode decomposition of numerical and experimental data. Journal of Fluid Mechanics 656, 5 (2010).

[21] E. Ferrer, , J. J. de Vicente, and E. Valero, Low cost 3D global instability analysis and flow sensitivity based on dynamic mode decomposition and high-order numerical tools. International Journal for Numerical Methods in Fluids 76, 15 (2014).

[22] S. Chandrasekhar. Hydrodynamic and Hydromagnetic Stability. Oxford Univ. Press, London, UK, 1968.

[23] Niels G. Jacobsen, , David R. Fuhrman, and Jørgen Fredsøe, A wave generation toolbox for the open-source CFD library: OpenFoam@. International Journal for Numerical Methods in Fluids 70, 1073-1088 (2011).

[24] Jie Zeng, and George Constantinescu, Flow and coherent structures around circular cylinders in shallow water. Physics of Fluids 29, 1073-1088 (2017).

[25] M. Heidari, , Ram Balachandar, , Vesselina Roussinova, and Ronald M. Barron, Characteristics of flow past a slender, emergent cylinder in shallow open channels. Physics of Fluids 29, 1073-1088 (2017).

[26] James H. Duncan, The breaking and non-breaking wave resistance of a two-dimensional hydrofoil. Journal of Fluid Mechanics 126, 507-520 (1983) 